\title{
Effect of Gramicidin S on the Dipalmitoylphosphatidyl- glycerol Thermotropic Phase Transition in DPPG/GS Systems: A Mathematical Approach
}

\author{
Cristina Stan \\ C. P. Cristescu \\ Department of Physics, Polytechnic University of Bucharest, \\ Bucharest, Romania

\section{Feride Severcan} \\ Department of Biology, Middle East Technical University of Ankara, \\ Ankara, Turkey
}

\section{Dana Dorohoi}

Faculty of Physics, Al. I. Cuza University of Iassy, Iassy, Romania

\begin{abstract}
We present a mathematical approach to the experimental data recorded via Fourier transform-infrared spectroscopy regarding the influence of the concentration of the antimicrobial peptide gramicidin $S(G S)$ on the thermotropic phase transition of the dipalmitoylphosphatidyl-glycerol (DPPG) lipid bilayer membrane in DPPG/GS systems. The model is based on the influence of the GS concentration on the parameters of a nonlinear damped oscillator, which models the $\mathrm{CH}_{2}$ symmetric stretching band.
\end{abstract}

Keywords: DPPG; FTIR spectra; GS; lipid membrane; phase transition

\section{INTRODUCTION}

The nature of phospholipid-peptide interactions influences the mechanism of membrane penetration by some peptides, such as antibiotics. When these interactions have an electrostatic nature [1], such as in the case of dipalmitoylphosphatidyl-glycerol (DPPG) (see the structural formula in Fig. 1a) and gramicidin S (GS) (see the conformational formula in Fig. 2), membrane destabilization can occur by the formation of

Address correspondence to Dana Dorohoi, Al. I. Cuza University of Iassy, Faculty of Physics, 11 Carol Blvd., 700506 Iassy, Romania. E-mail: ddorohoi@uaic.ro 


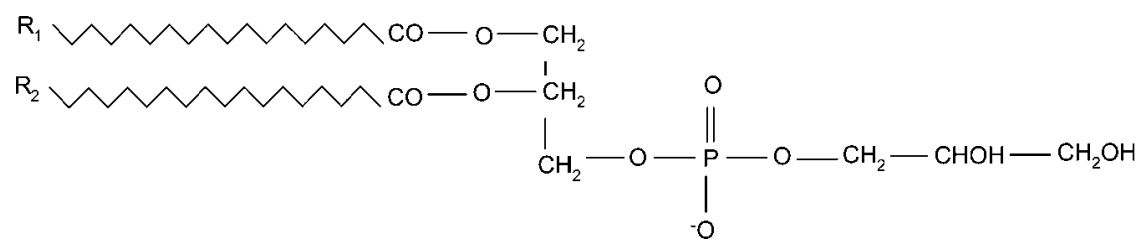

FIGURE 1 Structural formula of DPPG phospholipids.

pores or by a detergent-like mechanism [2,3], favoring the leakage of the cellular content [4].

DPPG [1] is an amphionic phospholipid in which two hydrophobic fatty acid chains are separated from a charged head group by a glycerol backbone. DPPG model membranes are structured as bilayers, with the interior fatty acid chains oriented in parallel to each other and the phospholipid heads facing out in contact with water. The stability of the DPPG model membranes is assured by maximization of hydrophobic interactions and by minimization of the hydrophilic ones. The strong interactions of the hydrophobic core are essential for the stability of bilayers as shown by the fact that the phase-transition temperature in saturated phospholipids increases with the acyl chain length [1].

Gramicidin $\mathrm{S}[5,6]$ is a very active anti-microbial and hemolytic peptide with a plated $\beta$-sheet structure, cyclo\{(Val-Orn-Leu-D-Phe-Pro $\left.)_{2}\right\}$ stabilized by four intramolecular hydrogen bonds between the two Leu and two Val residues [7,8]. The structure of the GS-component amino acids is shown in Fig. 3. Among the amino acid sequences, Orn has a basic character, and Val and Leu have hydrophobic characters [9]. Thus, the planar molecules of GS are amphiphilic; they have

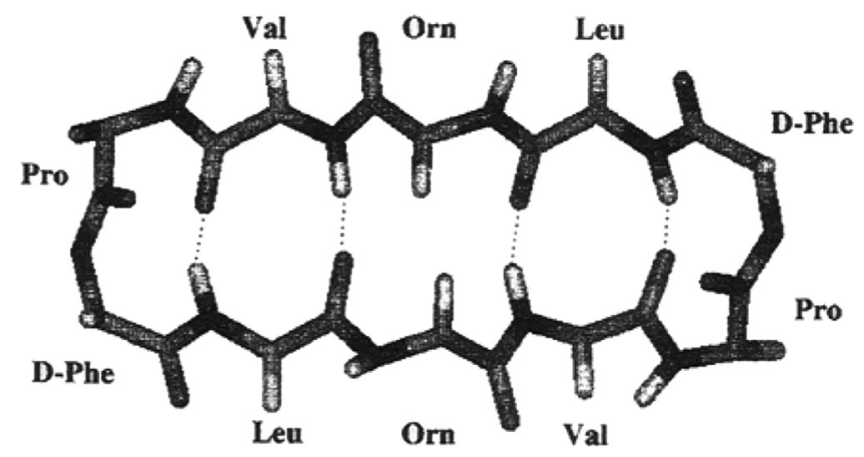

FIGURE 2 Conformational formula of GS. 


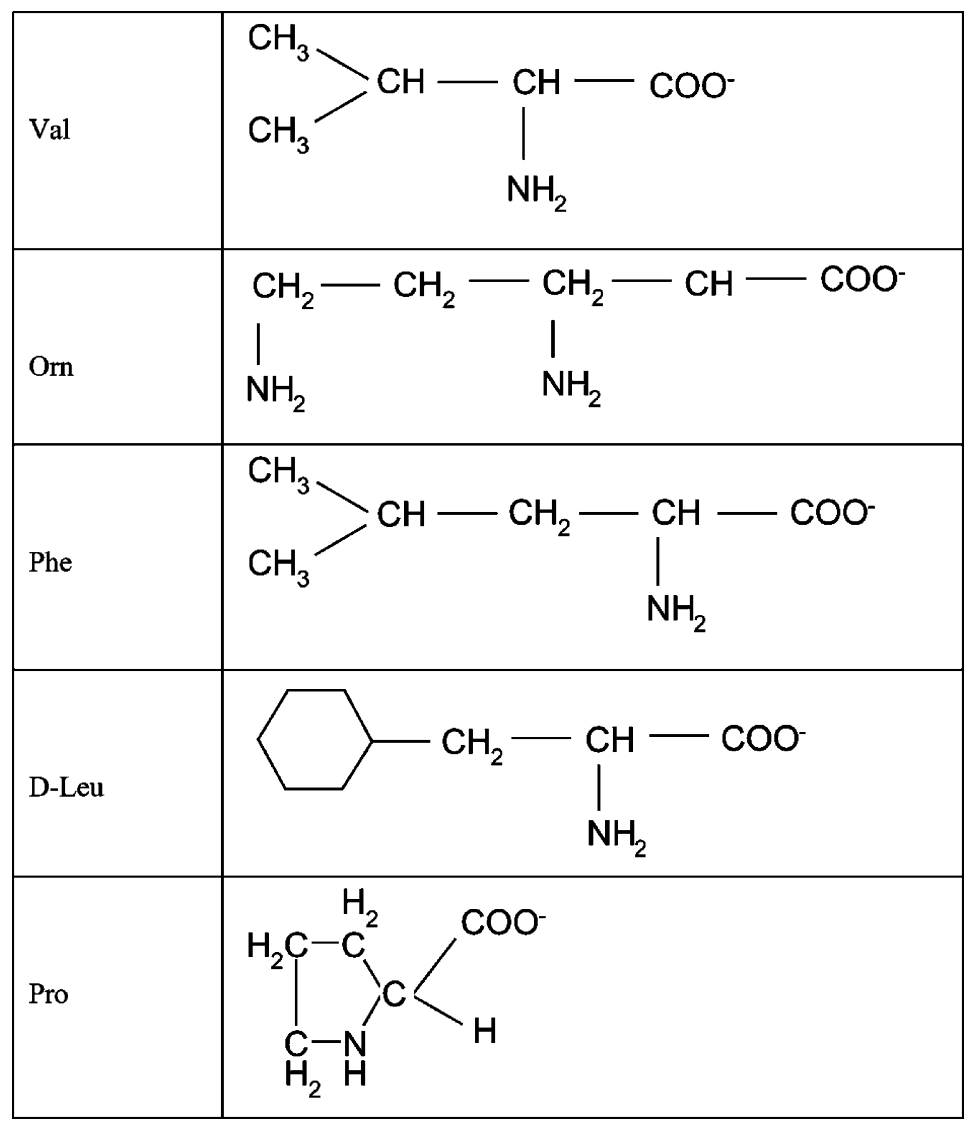

FIGURE 3 Structure of the amino acids in the GS molecule.

four Leu and Val side chains on the hydrophobic part and two polar positively charged Orn side chains and two D-Phe rings on the hydrophilic part.

NMR, X-ray, and MD studies indicate that the backbone adopts an antiparallel 3 -sheet with two Type II' $\beta$-turns in various GS solutions [10]. As consequence, in various media, the GS structure is amphipathic, with the hydrophobic side chains on one side of the molecule and the hydrophilic ones on the other. This provides a logical geometry for the interaction of the lipid membrane with the polar side of GS at the lipid/water interface and for the nonpolar side interaction with the lipid tails. There is considerable experimental evidence to support the GS conformation as described. 
The interaction of the positively charged Orn chains of GS with the negative part of the phospholipid backbone can induce a curvature stress modification in the hydrophobic part of the model membrane. When the curvature stress reaches a critical value, membrane destabilization occurs, its fluidity increases, the order degree decreases, and a liquid-crystalline phase begins. In Refs. [7,8], it is suggested that the ability of the GS to induce localized regions of high curvature stress in the lipid bilayers may be relevant to the mechanism by which this peptide disrupts the cell membrane.

Temperature variation induces changes in the degree of order of the model membrane [1,8]. At low temperatures, DPPG model membranes are structured in lamellar symmetric bilayers, forming a gel phase with a high degree of orientational order. At high temperatures, they pass into a less ordered, liquid-crystalline phase. This transition appears around $41^{\circ} \mathrm{C}$ and is illustrated in Fig. 4. The transition of the model membrane from the gel phase to the liquid-crystalline phase is a sudden process characterized by a main phase-transition temperature, $T_{m}$. At temperatures hotter than $T_{m}$, the lipids exist as a solidlike gel where the acyl chains are packed tightly against each other. At temperatures cooler than $T_{m}$, the lipids are in a liquid-like phase, which is in fact a liquid-crystalline phase. The acyl chains are disordered. Also, at temperatures hotter than $T_{m}$, rapid lateral diffusion of lipids (and proteins) occurs in the plane of the membrane.

The value of $T_{m}$ depends on the nature of the constituents and on their concentration in the model membrane [7,11-17].

When the temperature is decreased, the system's behavior is different and the DPPG/GS exhibits hysteresis phenomena.

The model membrane study in the neighborhood of $T_{m}$ can discover supplementary information on the membrane organization and on the membrane destabilization mechanism by addition of GS.

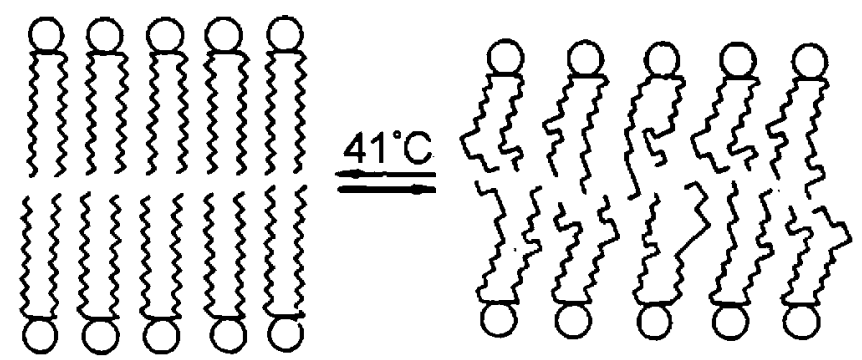

FIGURE 4 Illustration of phase transition of a phospholipid model membrane induced by temperature variation. 
Our interpretation of the experimental results is based on the hypothesis that the influence of GS concentration on the behavior of the nonlinear damped oscillator (which models the $\mathrm{CH}_{2}$ symmetric stretching mode) is different for the gel and the liquid-crystalline phases of the DPPG/GS systems.

\section{MATERIALS AND METHODS}

DPPG membranes and gramicidin S, purchased from Sigma Chemical Co., St. Louis, USA, were used without purification and stored at temperatures below $0^{\circ} \mathrm{C}$.

Multilamellar DPPG liposomes were obtained [11,14,15] by vortexing DPPG thin films in excess $\mathrm{Na}_{2} \mathrm{HPO}_{4}$ buffer for 20 min at $66^{\circ} \mathrm{C}$. The liposomes were incubated in a shaking water bath for $1 \mathrm{~h}$ at a temperature around $45^{\circ} \mathrm{C}$. The DPPG/GS systems were obtained from a stock solution of GS in ethanol. Multilamellar GS-containing vesicles were obtained using the same procedure followed for the DPPG vesicle preparation proposed in Refs. [14,15].

Fourier transform infrared (FTIR) spectra were registered using a Bomem 157 FTIR spectrometer. The interferograms were averaged for 100 scans. The samples were investigated in a large temperature range with temperature increasing from 26.5 to $61.1^{\circ} \mathrm{C}$ and decreasing from 61.1 to $26.5^{\circ} \mathrm{C}$. After preliminary experiments, we found a rate small enough to ensure cvasistatic processes.

An Unicam Specac digital temperature controller unit with a thermocouple located around the edge of the cell window was used for temperature monitoring. Each spectrum was recorded after $15 \mathrm{~min}$ of temperature stabilization to make sure that the sample temperature was accurately displayed by the digital controller.

Aqueous absorption caused by the added buffer in the samples was subtracted from the digitally observed spectra of investigated samples before analysis.

\section{RESULTS AND DISCUSSION}

The bandwidth of the $\mathrm{CH}_{2}$ symmetric stretching band was measured using the subtracted FTIR spectra for the spectral range $2800-3000 \mathrm{~cm}^{-1}$.

With increasing temperature, two changes in FTIR spectra were observed: a shift of the bands corresponding both to the symmetric and asymmetric stretching of the $\mathrm{CH}_{2}$ acyl chains toward high frequencies and an increase in the bandwidth. The bandwidth jumps near the main phase transition. These modifications are analyzed in the literature [7,11-16] for various types of phospholipids. We give a 
physical explanation of these modifications by analyzing the bandwidth values at different temperatures and different GS concentrations relevant for the dynamics of the chemical bonds implied in the IR bands' appearance. From the preliminary observations, the symmetric stretching band of $\mathrm{CH}_{2}$ appears sensitive to the temperature and GS concentration changes; consequently we analyzed the bandwidth of the symmetrical stretching vibration.

The plot of the experimental data of the $\mathrm{CH}_{2}$ symmetric stretching bandwidth versus increasing temperature near the main phase transition in the neighborhood of $42^{\circ} \mathrm{C}$ as shown in Fig. 5. Another jump is observed at values below $42^{\circ} \mathrm{C}$ for decreasing temperature, generating hysteresis cycles. The two hysteresis cycles represent 0\% GS concentration (solid symbols) and 10\% GS concentration (open symbols), the highest considered in the present work. The curves for intermediate values are omitted for clarity's sake.

Within the range of experimental errors, for all the values of the concentration of GS between $0 \%$ and $10 \%$, the bandwidth jumps for increasing temperature take place at the same temperature $T_{m}$, and they have the same value, as demonstrated by the curves marked with squares in Fig. 5. The phase-transition temperature $T_{m}$ could be defined as the temperature corresponding to the midpoint of the bandwidth shift between the gel phase and the liquid-crystalline phase. At this temperature, one can suppose that gel and liquid-crystalline phases coexist in equal proportions in the phospholipid membrane.

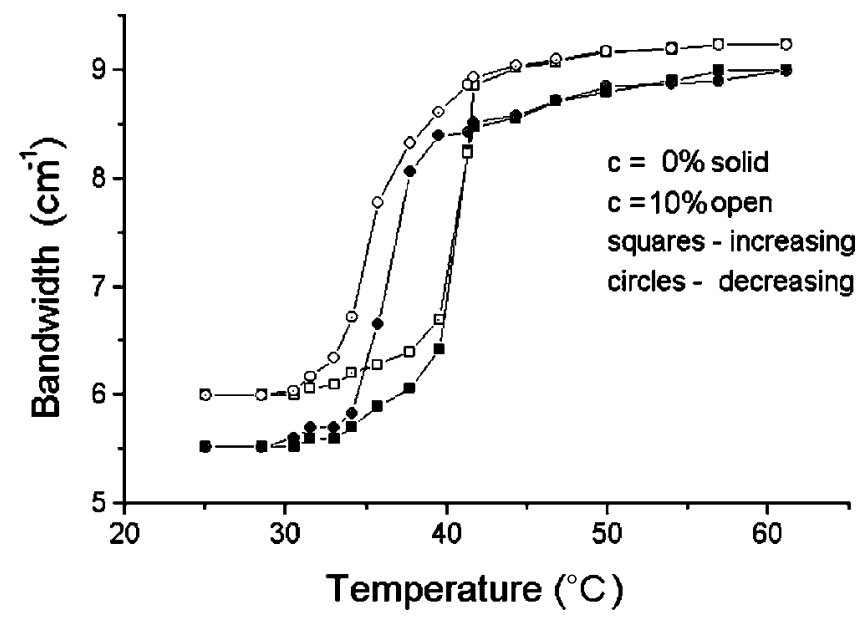

FIGURE 5 Hysteresis cycles in bandwidth versus temperature curves for zero GS concentration (solid marks) and 10\% GS concentration (open marks). 
The temperature at which the phase transition takes place is given by the maximum of the derivative of the bandwidth versus temperature curve. These curves have been computed by numerical differentiation of the experimental data of Fig. 5 and are shown in Fig. 6. They correspond to $0 \%$ (continuous line) and 10\% (broken line) GS concentration. The curves on the right side of Fig. 6, corresponding to increasing temperature, are highest at approximately the same temperature and have the same full width at high maximum (FWHM). The curves on the left side of Fig. 6, corresponding to decreasing temperature, have different maximums and different FWHM. The ratio of the FWHM for these curves is 1.35 . The transition curves for decreasing temperature should be asymmetric, in contradiction with the increasing temperature bandwidth curves. This asymmetry is visible on the dotted-line curve.

For all GS concentrations in this study, in the plot of the bandwidth for decreasing temperature, the jumps occur at different temperatures. The higher the concentration, the lower the temperature of the transition. Also, we observe a change in the magnitude of the jump, defined as the rectilinear part of the curve for decreasing temperature, which diminishes

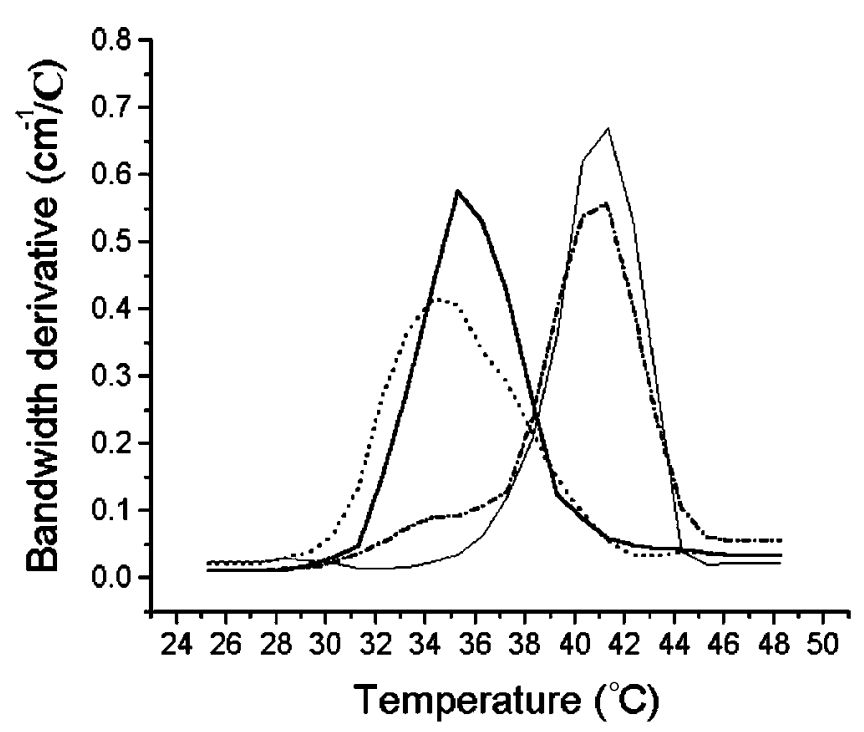

FIGURE 6 Numerical derivatives of the experimental curves in Fig. 1. The curves on the right with the same FWHM and the same position of the maximum correspond to increasing temperature, whereas the curves on the left correspond to decreasing temperature. The curve with the larger FWHM and lower temperature maximum (dotted line) represents $10 \%$ GS, and the curve with the heavy line represents $0 \%$ GS concentration. 


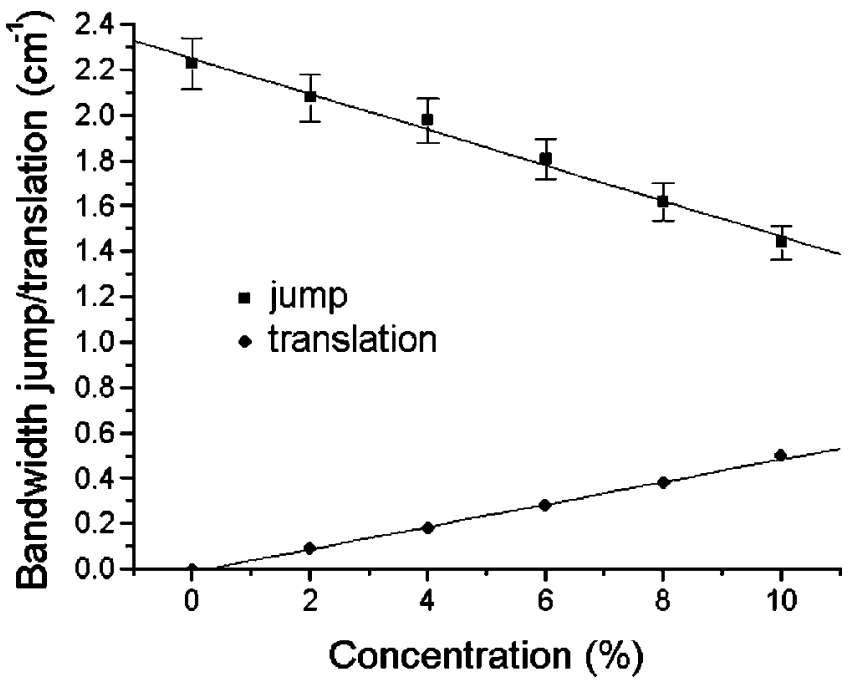

FIGURE 7 Magnitude of the bandwidth jump (squares) and the bandwidth translation (circles) versus GS concentration.

with increasing concentration. This behavior is clearly shown by the upper curve in Fig. 7. The experimental points can be fitted as a linear dependence; the error bars correspond to an estimated precision of $5 \%$.

Another clear change induced by the presence of GS is the translation of the bandwidth versus temperature curve toward higher values with increasing GS concentration. It is represented by the lower curve in Fig. 7. Globally, these effects are also visible in the dependence of the area of the hysteresis loop on the GS concentration shown in Fig. 8. The experimental points are well situated on a second-order curve as fitted by the equation written on Fig. 8.

These results are in agreement with measurements on the thermotropic phase behavior of similar systems using differential scanning calorimetry (DSC). On the bases of DSC studies, Prenner and his coworkers [17] assumed that GS in the bilayer is located at the polar/ apolar interface of liquid-crystalline bilayer, where it interacts primarily with the polar head and glycerol backbone regions of the phospholipid molecules and only secondarily with the lipid hydrocarbon chains.

\section{MATHEMATICAL APPROACH}

The model of nonlinear oscillators has been used extensively for interpretation of the behavior of a wide range of physical systems from 


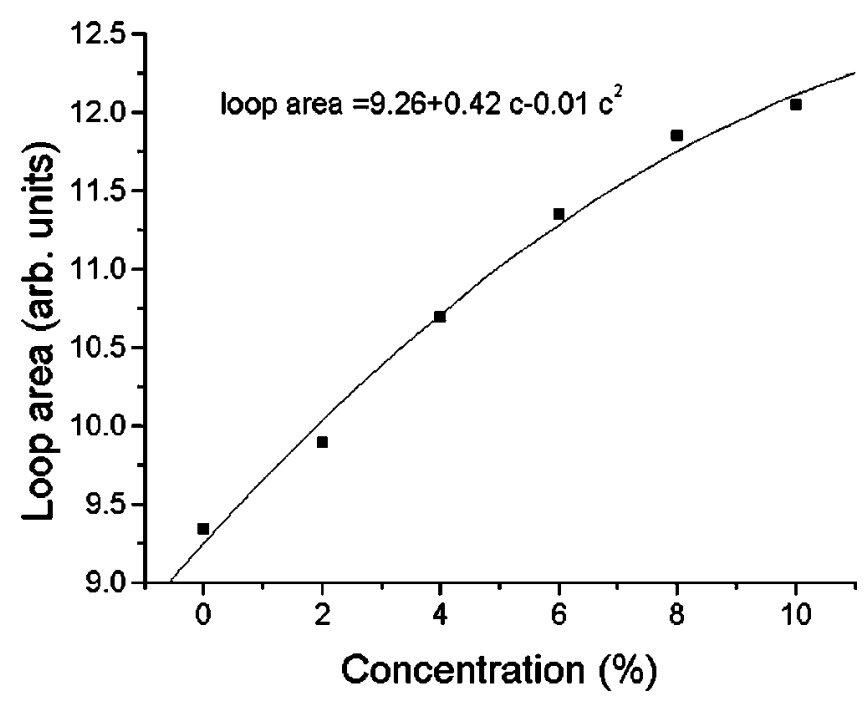

FIGURE 8 Area of the loop hysteresis curve versus GS concentration.

plasmas [18-20] and condensed matter [21] to DNA and neuronal dynamics [22,23].

The structure of lipid bilayers, liposomes, and the nature of the phase transition is covered in a wide array of literature [24-26]. The transition from gel to the liquid-crystalline phase involves a partial melting of the acyl chains while the spherical liposome remain intact. In the liquid-crystalline states, the acyl chains become mobile within the hydrophobic region of the liposome while the anchoring of the polar head groups prevents complete freedom of movement.

In Ref. [16], it was mentioned that the presence of GS appears to induce the formation of inverted cubic phases at lower temperatures in those lipids capable of forming such phases at higher temperatures in the absence of peptide. Also, GS increases the permeability both of the model and biological membranes and, at higher concentrations, causes membrane destabilization. Based on this information, we constructed a model of the experimental results that takes into consideration that the influence of the GS concentration on the behavior of a nonlinear damped oscillator modeling the $\mathrm{CH}_{2}$ symmetric stretching mode is different in the gel than in the liquid-crystalline phases of the DPPG/GS system. We admit that in the highly organized gel phase, the influence of the GS presence is mainly on the damping coefficient and less on the potential distribution, whereas in the less organized liquid-crystalline phase, besides influence on the damping 
coefficient, an important change in the potential distribution is induced. That a system characterized by a double well potential has a hysteresis-like characteristic was recognized as early as 1948 [27].

In our model, we consider a sixth-order potential in agreement with the space charge distribution along a bilayer, as obtained from X-ray diffraction patterns [28]:

$$
V(x)=\frac{1}{2} x^{2}-\frac{\beta}{4} x^{4}+\varepsilon x^{6}
$$

with $\beta>0, \varepsilon>0$, where $x$ stands for the inter atomic instantaneous distance normalized to the average value.

Because $\mathrm{x}<1$, the dynamics is restricted only in the central zone of the graph in Fig. 9 marked by the broken curve and shown enlarged in the inset in the same figure. The change induced by the GS concentration consists of a deepening of the central well as shown in Fig. 9 (inset) for two different values of the fourth-order coefficient $(\beta=0.100$ and $\beta=0.072)$ and the same sixth-order coefficient $\varepsilon=0.0002$. These values of the parameters are chosen simply for illustration of the behavior of the sixth-order potential (1) and the equivalence of its central part with a fourth-order potential [first two terms in Eq. (1)]. A posteriori, we find that these values give results of the model in reasonable agreement with the experiment.

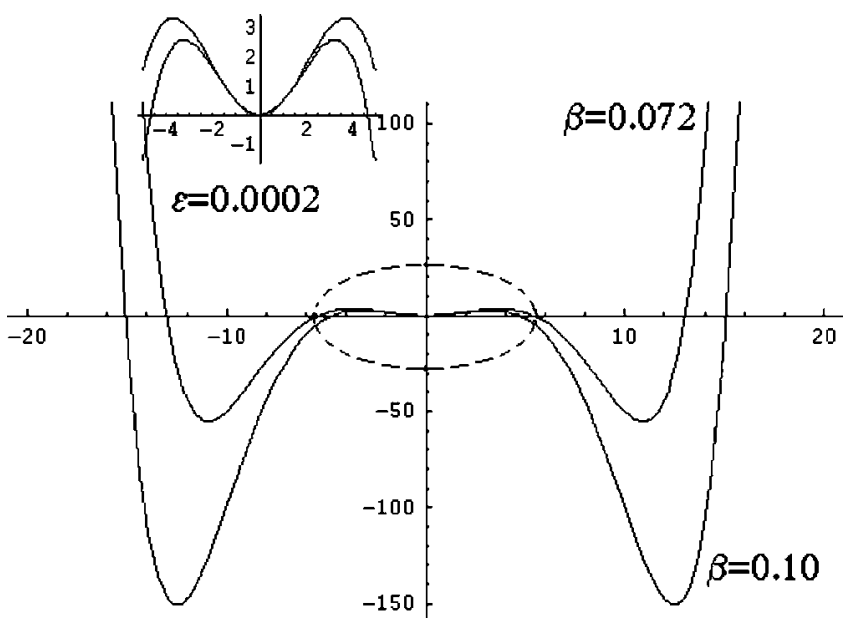

FIGURE 9 Potential curves showing the changes induced by increasing GS concentration on the parameters of the oscillator. The main effect is in on the small amplitude domain marked by a broken line circle and enlarged in the inset. 
Consequently, the effect of the GS presence in the DPPG/GS system can be modeled by a change in the behavior of a Duffing-type oscillator with a fourth-order potential, shown in the inset in Fig. 9 and described by the first two terms on the right side of Eq. (1).

The Duffing equation for the stretching motion subject to a dissipation $-\gamma \dot{x}$, and the harmonic driving $\cos \omega t$ is

$$
\ddot{x}+\gamma \dot{x}+x-\beta x^{3}=\cos \omega t
$$

We consider the dynamics of this system only for amplitudes below the value corresponding to the "escape or dissociation threshold"

$$
a_{t h}=\sqrt{\frac{1}{\beta}}
$$

It is well known that this system could show bistable behavior and that in the bistability zone chaotic behavior appears [28,29]. Good results for the behavior of the Duffing system (2) are obtained using the slowly varying amplitude (SVA) approximation. We write

$$
x(t)=\frac{1}{2}\left(a(t) \exp [i \omega t]+a^{*}(t) \exp [-i \omega t]\right)
$$

and assume that $a(t)$ is slowly varying, that is, $|\ddot{a}(t)|<<\omega^{2}|a(t)|$.

Equation (2) reduces to the approximate form

$$
(\gamma+2 i \omega) \dot{a}+\left(\frac{1-\omega^{2}+i \gamma \omega-3|a|^{2} \beta}{4}\right) a=1
$$

This equation has steady-state solution $\bar{a}$ satisfying the fixed-point condition

$$
\bar{a}\left(\frac{1-\omega^{2}+i \gamma \omega-3|\bar{a}|^{2} \beta}{4}\right)=1
$$

In the SVA approximation, the steady-state solution $\bar{a}$ replaces $a(\mathrm{t})$ in Eq. (4). Equation (6) is equivalent to the following (real) cubic equation for $|\bar{a}|^{2}$ :

$$
|\bar{a}|^{2}\left[\omega^{2} \gamma^{2}+\left(\frac{1-\omega^{2}-3|\bar{a}|^{2} \beta}{4}\right)^{2}\right]=1
$$

The solution of this equation is shown in Fig. 10a for $\gamma=0.47$, $\beta=0.100$, and in Fig. 10b for $\gamma=0.47, \beta=0.072$. We consider the interval $B$ shown in Fig. 10a as an additional bandwidth jump such that the measured bandwidth is modeled by $B_{0}-B$, where $B_{0}$ is a constant proportional to the bandwidth jump with $0 \%$ GS concentration. 


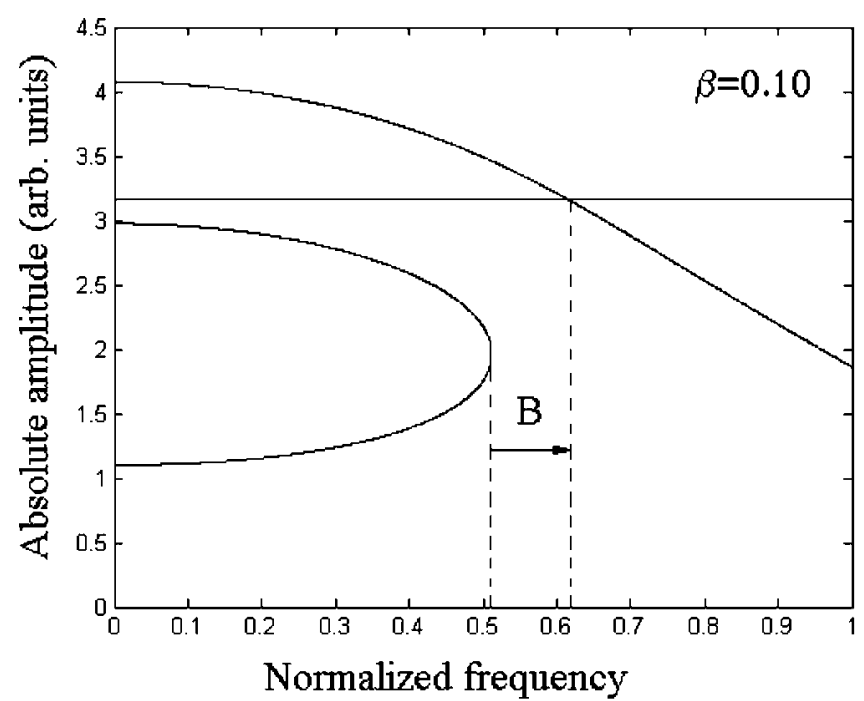

(a)

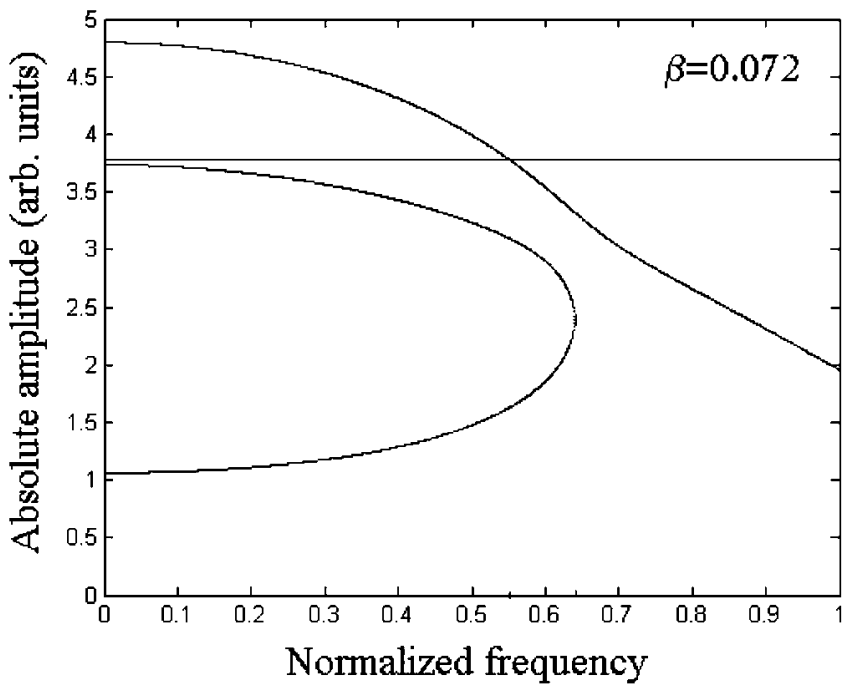

(b)

FIGURE 10 Absolute value of the amplitude of the nonlinear damped oscillator versus driving frequency in the rotating-wave approximation showing the adopted definition of the bandwidth (B): a) $\beta=0.100$; and b) $\beta=0.072$. 


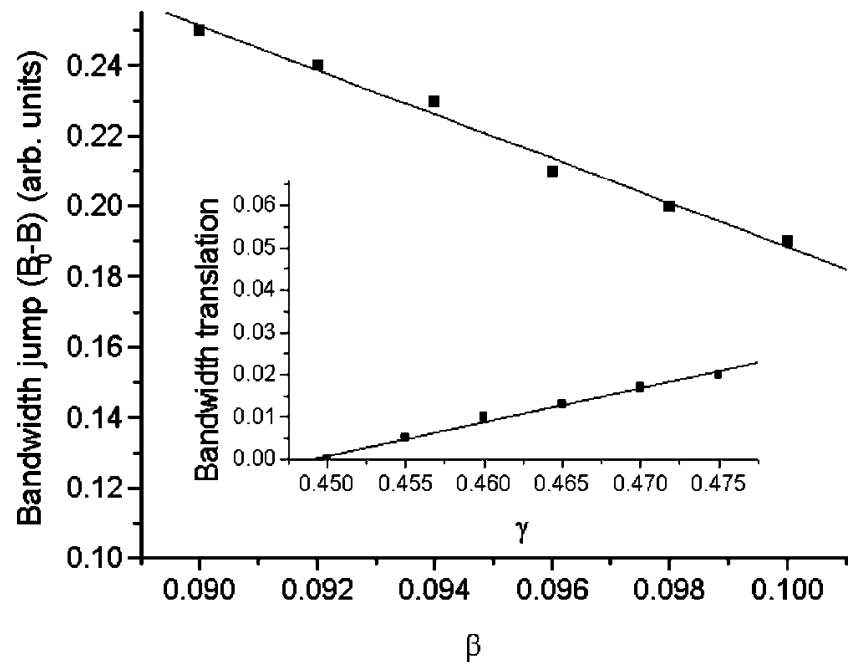

FIGURE 11 Dependence of the adopted bandwidth $B_{0}-B$ (where $B_{0}=0.3$ is a reference value) versus $\beta$ and of the displacement of the lower limit of the $B$ interval versus the damping coefficient $\gamma$ (as inset).

The linear decrease of $B_{0}-B$ with the increase of the $\beta$ coefficient, in agreement with the upper curve on Fig. 7, suggests that our hypothesis on the linear dependence of $\beta$ on the GS concentration is valid.

The modeled bandwidth jump has a linear decrease with $\beta$, as shown in Fig. 11. On the same figure, the dependence of the bandwidth translation modeled by the displacement of the point where two of the solutions of Eq. (7) became complex conjugate is shown in the inset. The linear change of this translation is also in agreement with the experimental behavior shown by the lower line on Fig. 7 .

With reference to the decreasing temperature curve in Fig. 5, the slower change of the slope in the upper neighborhood of the transition jump as compared to the slope variation in the lower bending zone might be interpreted as a consequence of a temperature-dependent relationship between $\beta$ and $\gamma$.

\section{CONCLUSIONS}

The results of this work show that the influence of gramicidin $\mathrm{S}$ on the dipalmitoylphosphatidyl-glycerol phase transitions in DPPG/GS systems, for concentrations up to $10 \%$, is more visible on the decreasing branch of the bandwidth versus temperature hysteresis loop. It mainly consists of three effects: a gradual decreasing in the magnitude of the 
bandwidth jump with increasing concentration, a translation of the whole hysteresis loop toward higher values of the bandwidth, and an asymmetry of the decreasing temperature branch of the hysteresis loop.

An increasing quantity of GS results in a change in the memory of the DPPG/GS system consisting of the persistence of the liquidcrystalline phase to lower temperatures than in the absence of GS.

We propose a computational analysis based on the hypothesis that the modification in the damping parameter and in the shape of the potential of a nonlinear oscillator could model the experimentally observed effects induced by increasing GS concentration. The results are in satisfactory qualitative agreement with our experimental data.

\section{ACKNOWLEDGMENTS}

The partial support of this study by NATO TUBITAK Committee of Turkey in the NATO PC-B Program is gratefully acknowledged by Dana Dorohoi.

\section{REFERENCES}

[1] Marsh, D. (1983). "Biomembranes". In: Supramolecular Structure and Function, Pifat, G. \& Herak, J. N. (Eds.), Plenum Press: New York, 127-178.

[2] Epand, R. M. (1998). Biochim. Biophys. Acta, 1376, 353-368.

[3] Lohner, K. \& Prenner, E. J. (1999). Biochim. Biophys. Acta, 1462, 141-156.

[4] Katsu, T., Kobayashi, H., Hirota, T., Fuita, Y., Sato, K., \& Hagay, U. (1987). Biochim. Biophys. Acta, 899, 57-63.

[5] Gause, G. F. \& Brashnikova, M. G. (1944). Nature, 154, 703.

[6] Izumiya, N., Kato, T., Aoyaga, H., Waki, M., \& Kondo, M. (Eds). (1979). Synthetic Aspects of Biologically Active Cyclic Peptides: Gramicidiu $S$ and Tyrocidines, Halsted Press: New York.

[7] Prenner, E. J., Lewis, R. N. A. H., \& Kodejewski, L. H. (1999). Biochim. Biophys. Acta, 1462, 201-221.

[8] Prenner, E. J., Lewis, R. N. A. H., Kodejewski, L. H., Flach, R. S., Mendelson, R., Hodges, R. S., \& Elhaney, R. N. (1999). Biochim. Biophys. Acta, 1417, 211-223.

[9] Lenhinger, A. (1975). Biochemistry, Worth Publishers: New York.

[10] Jones, C. R., Sikakana, C. T., Hehir, S., Kuo, M.-C., \& Gibbons, W. A. (1978). Biophys. J., 24, 815-832.

[11] Severcan, F., Durmus, H. O., Eker, F., Haris, P. I., \& Akinoglu, B. G. (2000). Talanta, 53, 205-211.

[12] Severcan, F., Tokmak, S., Agheorghiesei, C., \& Dorohoi, D. (2002). Annals of Univ. Al. I. Cuza, Iassy, Section Chemistry, Tom X, 259-265.

[13] Geith, M. (1999). PhD Thesis, METU, Ankara, Turkey.

[14] Severcan, F., Kazanci, N., Baycal, U., \& Suzer, S. (1995). Biosci Rep., 15, 221.

[15] Akinoglu, B. G., Gheith, M., \& Severcan, F. (2001). J. Molec. Struct., 565-566, 281-285.

[16] Stan, C., Cristescu, C. P., Severcan F., \& Dorohoi, D. (2004). Rev. Roum. Chim., 49(9), 777-782. 
[17] Prenner, E. J., Lewis, R. N. A. H., \& McElhaney, R. N. (2001). Biophys J., 80(4), 1873-1890.

[18] Stan, C., Cristescu, C. P., \& Alexandroaei, D. (2002). Contrib. Plasma Phys., 42, 81-89.

[19] Cristescu, C. P., Stan, C., \& Alexandroaei, D. (2002). Phys Rev., E66, 016602-016608.

[20] Klinger, T., Piel, A., Seddighi, F., \& Wilke, C. (1993). Phys. Lett., A182, 312.

[21] Peyrand, M. \& Bishop, A. R. (1989). Phys. Rev. Lett., 62, 8755-8758.

[22] Oprisan, S. A. \& Canavier, C. C. (2002). Neural Comp., 14, 1027-1057.

[23] Othmer, H. G. (Ed.), (1986). Nonlinear Oscillation in Biology and Chemistry, Springer-Verlag: New York.

[24] Marshall, A. G. (1978). Biophysical Chemistry: Principles, Technique and Applications, Wiley: New York.

[25] Holtzer, A. J. (1995). J. Phys. Chem., 99, 13048-13049.

[26] Ragone, R. \& Colonna, G. (1995). J. Phys. Chem., 99, 13050-13054.

[27] Stoner, E. C. \& Wohlfarht, E. P. (1948). Philos. Trans. R. Soc. London, A240, 599-607.

[28] Huberman, B. A. \& Crutchfield, J. P. (1979). Phys. Rev. Lett., 43, 1743-1745.

[29] Lewis, R. N. A. H., Winter, I., Kriechbaum, M., Lohner, K., \& McElhaney, R. N. (2001). Biophys J., 80(3), 1329-1342. 